\title{
Antibiotic Prescribing Practices for Catheter Urine Culture Results
}

\author{
Jonathan Chiu, G William Thompson, Thomas W Austin, Zafar Hussain, Michael John, \\ Anne Marie Bombassaro, Sarah E Connelly, and Sameer Elsayed
}

\begin{abstract}
Background: The literature suggests that positive results of catheter urine cultures frequently lead to unnecessary antimicrobial prescribing, which therefore represents an important target for stewardship.

Objective: To assess the appropriateness of antibiotic prescribing in response to the results of urine cultures from patients with indwelling urinary catheters.

Methods: This retrospective study was conducted at a tertiary care centre and involved adults with indwelling urinary catheters from whom urine specimens were obtained for culture. Patients with positive or negative culture results were identified from microbiology laboratory reports. The medical records of consecutive patients were screened to select a sample of 80 inpatients ( 40 per group). Abstracted patient histories were independently evaluated by an expert panel of 3 infectious diseases consultants blinded to the decisions of prescribers and of fellow panelists. The primary end point was concordance of each patient's treatment decision (with respect to the indication) between the expert panel (based on majority agreement, i.e., at least 2 of the 3 expert panelists) and the prescriber. The secondary end points were unnecessary days of therapy and selected outcomes over a predefined period after urine was obtained for culture.
\end{abstract}

Results: A total of 591 charts were screened to generate the targeted number of patients. Baseline demographic characteristics were comparable for the 2 groups, except antibiotic exposure before urine collection was significantly more frequent for the group with negative culture results. The treatment decision was concordant in 40\% (16/40) of the patients with a positive culture result and $85 \%(34 / 40)$ of those with a negative culture result $(p<0.001)$. The most common reason for discordance was administration of antibiotics when not indicated ( 23 of 24 patients with a positive result and 5 of 6 patients with a negative result), which accounted for 165 and 32 unnecessary days of therapy per 1000 inpatient-days, respectively $(p<0.001)$. Adverse effects occurred in 2 of the 23 patients with a positive result who received antibiotics that were not indicated.

Conclusions: Appropriateness of antibiotic prescribing, as measured by concordance of decisions between the expert panel and prescribers, was more common among patients with negative urine culture results than among those with positive results. However, there is an opportunity to improve prescribing for both groups through antimicrobial stewardship initiatives. Unnecessary days of therapy and adverse effects were more common in patients with a positive culture result.

Key words: antibiotic, antimicrobial, asymptomatic bacteriuria, catheter, prescribing

\section{RÉSUMÉ}

Contexte : Selon la littérature, des résultats positifs à des cultures d'échantillons d'urine prélevés à partir d'une sonde vésicale entraînent souvent la prescription inutile d'antibiotiques; par conséquent, cette situation représente un objet important de gestion responsable des antimicrobiens.

Objectif : Évaluer la pertinence de la prescription d'antibiotiques sur la base de résultats des cultures d'échantillons d'urine prélevés chez les patients porteurs d'une sonde vésicale à demeure.

Méthodes : La présente étude rétrospective a été menée dans un établissement de soins tertiaires auprès de patients adultes porteurs d'une sonde vésicale à demeure chez qui on a prélevé des échantillons d'urine à des fins de culture. Les patients avec des résultats de culture positifs ou négatifs ont été repérés à partir des rapports de microbiologie. Les dossiers médicaux d'une série consécutive de patients ont été examinés afin de choisir un échantillon de 80 patients hospitalisés ( 40 par groupe). Les historiques abrégés des patients ont été évalués indépendamment par un panel d'experts composé de trois consultants en maladies infectieuses qui ignoraient la décision des prescripteurs et les résultats des évaluations de leurs collègues panélistes. Le paramètre d'évaluation principal était la concordance de la décision thérapeutique (quant à l'indication) pour chaque patient entre le panel d'experts (selon un accord majoritaire, c.-à-d. au moins deux des trois panélistes experts) et le prescripteur. Les paramètres d'évaluation secondaires étaient le nombre de jours de traitement inutiles et certains résultats cliniques sur une période prédéterminée après l'obtention d'un échantillon d'urine à des fins de culture.

Résultats : Un total de 591 dossiers de patients ont été analysés afin d'obtenir le nombre cible de patients pour l'étude. Les caractéristiques démographiques initiales étaient comparables dans les deux groupes, sauf l'exposition aux antibiotiques avant le prélèvement d'urine qui était significativement plus fréquente dans le groupe de patients ayant obtenu des résultats de culture négatifs. La décision thérapeutique concordait pour $40 \%(16 / 40)$ des patients ayant obtenu des résultats positifs à la culture d'urine et pour $85 \%$ (34/40) de ceux qui avaient obtenu des résultats négatifs $(p<0,001)$. La raison la plus fréquente expliquant la discordance entre les décisions thérapeutiques était l'administration non indiquée d'antibiotiques, une circonstance observée chez 23 patients sur 24 ayant obtenu des résultats positifs et 5 patients sur 6 ayant obtenu des résultats négatifs. Ces cas ont contribué respectivement à un total de 165 et de 32 jours de traitement inutiles par 1000 journées-patients hospitalisés $(p<0,001)$. Des effets indésirables sont survenus chez deux des 23 patients ayant obtenu un résultat positif et reçu des antibiotiques non indiqués.

Conclusions : La pertinence de la prescription d'antibiotiques, telle qu'elle a été mesurée en fonction de la concordance des décisions entre 
le panel d'experts et les prescripteurs, était plus fréquente dans le cas des patients ayant obtenu des résultats négatifs à la culture d'urine que chez ceux dont ces résultats étaient positifs. Cependant, des mesures pour la gestion responsable des antimicrobiens pourraient améliorer la prescription dans les deux groupes. Le nombre de jours de traitement inutiles et les effets indésirables étaient plus fréquents chez les patients ayant obtenu des résultats positifs à la culture.

Mots clés : antibiotique, antimicrobien, bactériurie asymptomatique, sonde, prescription

[Traduction par l'éditeur]

\section{INTRODUCTION}

$\mathrm{U}$ rinary catheters have been used for centuries on an intermittent or indwelling basis as a treatment for urinary retention. ${ }^{1}$ Generally accepted indications for indwelling urinary catheterization include clinically significant urinary retention or incontinence, requirement for accurate monitoring of urine output, prolonged surgical procedures, and selected urologic and gynecologic procedures (for which catheterization is used in the perioperative period). ${ }^{1,2}$ However, catheterization increases the risk of introducing bacteria into the normally sterile urinary tract, which results in catheter-associated bacteriuria (asymptomatic in most patients)..$^{1-3}$ The duration of catheterization is a significant risk factor for the development of bacteriuria, the acquisition rate being $3 \%-10 \%$ per day, with nearly all patients having bacteriuria by 30 days. ${ }^{1,2,46}$ The Infectious Diseases Society of America (IDSA) does not recommend routine screening for and treatment of asymptomatic bacteriuria in catheterized patients, except pregnant women and patients undergoing traumatic genitourinary procedures with associated mucosal bleeding. ${ }^{2}$

Catheter-associated asymptomatic bacteriuria has been described by the IDSA as "a tempting target for physicians who have a low threshold for using antimicrobials", ${ }^{2}$ and this indication has been reported to result in the unnecessary use of antimicrobials. ${ }^{7-10}$ Silver and others ${ }^{7}$ described the pattern of antimicrobial use in response to urine culture results in a cohort of hospitalized patients, regardless of catheterization, and found that 43 (64\%) of 67 patients with asymptomatic bacteriuria were treated. A similar result (antimicrobial treatment for 41 [65\%] of 63 patients) was observed in the pre-intervention population in a study assessing the effect of education on unnecessary antibiotic use for asymptomatic bacteriuria in patients with and without urinary catheters. ${ }^{8}$ In a small prospective study at a Canadian tertiary care hospital, Dalen and others ${ }^{9}$ determined that antimicrobials had been prescribed for $52 \%$ of catheterized patients with asymptomatic bacteriuria and therefore management was inappropriate. Cope and others $^{10}$ reported that treatment was administered in $32 \%$ of episodes of catheter-associated asymptomatic bacteriuria in a tertiary care hospital in the United States.

Antibiotic prescribing practices in response to the results of catheter urine cultures have not previously been described at the authors' institution. The objective of this retrospective study was to assess the appropriateness of antibiotic prescribing in response to such results. The primary end point was concordance of the treatment decision, with respect to the indication for therapy, for patients with positive or negative urine culture results. Concordance was determined between an expert panel (based on retrospective assessment of medical records) and the prescribers who provided care during the hospital stay. Secondary end points included the number of unnecessary days of antibiotic therapy and other predefined outcomes.

\section{METHODS}

This retrospective study was conducted at the Victoria Hospital site of the London Health Sciences Centre, in London, Ontario, a tertiary care teaching hospital. Approval for the study was obtained from the Office of Research Ethics governing the institution.

\section{Enrolment}

Adults at least 18 years of age who had been admitted to an inpatient ward at the study site over a 6-month period (June 1 to November 30, 2010) and for whom a urine specimen from an indwelling catheter had been collected for culture were eligible for inclusion. The source population was identified from a chronological report generated by the microbiology laboratory of patients for whom catheter urine cultures were performed over the study period. This population was screened for inclusion by a 2 -stage process. The initial screening involved cross-checking the microbiology report against a list generated by the Health Records Department that included inpatients at least 18 years of age who had been admitted to the study site over the same period. This initial screen excluded patients who met the criteria specified in Appendix 1, Part 1. The second screening, which involved manual chart review for the exclu- 
sion criteria specified in Appendix 1, Parts 1 and 2, was applied to the remaining patients (consecutively by date of admission). Screening continued until a convenience sample of 80 inpatients, 40 with a positive result and 40 with a negative result, had been enrolled. Any individual patient could be enrolled only once, regardless of the number of admissions or cultures in the 6-month study period.

\section{Definitions and End Points}

A positive culture result was defined as the presence of 1 or 2 species of micro-organisms in any quantity; patients with mixed bacterial growth (more than 2 micro-organisms) were excluded (see Appendix 1, Part 1). A negative culture result was defined as "no growth".

The primary end point, concordance, was defined as agreement in the treatment decision for each patient, with respect to indication for therapy, between a blinded expert panel, consisting of 3 infectious diseases consultants (G.W.T., T.W.A., S.E.), and the prescriber who treated the patient during the hospital stay. Each treatment decision was categorized as either concordant or discordant.

The secondary end points included the number of unnecessary days of therapy (total days and inpatient days only), along with other predefined outcomes. Unnecessary days of therapy referred to the receipt of at least one antibiotic dose on a day when therapy was not indicated according to the recommendations of the expert panel. The other predefined outcomes of interest were initiation of therapy beyond $48 \mathrm{~h}$ after the final microbiology report, retreatment, development of an antibiotic-related complication, and readmission to the study hospital because of a urinary indication (i.e., urine culture result and/or associated signs or symptoms) or a treatment complication.

\section{Data Collection}

A single investigator (J.C.) conducted a comprehensive review of each patient's health record to collect demographic and clinical characteristics, including duration of catheterization, prior and concurrent antibiotic and antipyretic use, past medical history, presenting signs and symptoms, diagnostic tests performed and their results, antibiotics prescribed, and duration of treatment. The Ontario Drug Benefit profile was reviewed, when available, to obtain further information on outpatient exposure to antibiotics. Data were manually transcribed onto a standardized form.

\section{Evaluation of Prescriber Treatment Decisions and Patient Outcomes}

Medication orders were reviewed from the time of urine collection until $48 \mathrm{~h}$ after the final microbiology report to determine if antibiotic therapy had been initiated. This period was referred to as the treatment decision observation period. The following protocol to evaluate prescribers' treatment decisions during this period was developed a priori. Patients for whom no antibiotics were prescribed during the treatment decision observation period were considered not to have received therapy. In cases where antibiotics were prescribed during the treatment decision observation period, the patients' medical records were reviewed to determine if the prescribed therapy was related to a urinary indication (i.e., urine culture result and/or associated signs or symptoms) or to an unrelated indication or culture result. If the medical record referred to the former, the patient was considered to have received therapy for a urinary indication. If reference was made to the latter, the patient was considered to have received therapy for a nonurinary indication.

In the absence of medical record documentation of the indication for any prescribed antibiotics, predefined assumptions (listed in Appendix 2) were used to evaluate the prescribers' treatment decisions. If the treatment indication was rated as undetermined according to these assumptions, the case was evaluated independently by a review panel. This panel was composed of 2 medical microbiologists (Z.H., M.J.) and an infectious diseases pharmacist (A.M.B.), who assigned by majority agreement an interpretation of the treatment received as being for a urinary or a non-urinary indication.

The predefined outcomes were assessed over a 7-day period. The starting date for this period was the end of the treatment decision observation period if no antibiotics were prescribed, the discharge date if discharge occurred before the end of the treatment decision observation period, or the last day of antibiotic therapy if such therapy was prescribed for a urinary indication. Follow-up of discharged patients was limited to information available from the study hospital, affiliated clinics, and the Ontario Drug Benefit medication profile.

\section{Evaluation of Concordance}

Information about each case was provided to each member of the expert panel for independent review. The information provided consisted of medical history, comorbidities, current clinical presentation, results of all diagnostic tests ordered up to $24 \mathrm{~h}$ after the collection of urine for culture, antibiotics administered over the 1-week period before collection of urine for culture, and concurrent use of antipyretics. The panel members were blinded to the therapy decisions made by the prescribers at the time of care. Treatment recommendations by the 3 experts were based on their clinical assessment of each case, rather than on predefined criteria. The treatment decision of the expert panel was determined by majority agreement (i.e., at least 2 of the 3 expert panelists) and was compared with the prescriber's actual treatment decision. 
For concordant cases, where the expert panel deemed that treatment was indicated and the prescriber had ordered treatment, one of the study investigators (J.C.) assessed the selected antibiotic for appropriateness on the basis of the culture and susceptibility report and the patient's allergy profile.

\section{Evaluation of Unnecessary Days of Therapy}

The number of unnecessary days of therapy for each group was expressed as a percentage of the number of days of therapy (inpatient plus outpatient days and inpatient days only). "Days of therapy" was defined as days of therapy received for inpatients and days of therapy prescribed for outpatients. The number of unnecessary days of therapy as an inpatient standardized to 1000 inpatient-days was also determined. For the purposes of this calculation, the dates of admission and discharge were counted as a single inpatient day.

\section{Statistical Analysis}

The mean and standard deviation were used to summarize normally distributed continuous variables, and the median and range were used to summarize skewed (i.e., not normally distributed) continuous variables. Percentages were calculated for categorical variables. The independent-samples $t$ test or, where applicable, the Mann-Whitney $U$ test, was used to compare differences in continuous variables between groups. The chi-square test was used to compare differences in categor- ical variables between groups. The level of agreement between treatment decisions of the expert panel and prescribers was determined with the kappa statistic. ${ }^{11}$ Statistical analyses were performed using SPSS Statistics, version 20 (IBM, Armonk, New York). Any $p$ value less than 0.05 was considered significant.

\section{RESULTS}

Reaching the target enrolment of 80 patients (40 in each group) required screening a total of 591 patient records, 277 for patients with a positive culture result and 314 for patients with a negative culture result. Outpatient status, admission to another hospital site, admission to the intensive care unit, immunosuppression (related to drug and/or disease), and cultures with mixed bacterial growth accounted for 215 (91\%) of the 237 exclusions among patients with a positive culture result. The first 4 of these criteria were also applicable to patients with negative culture results, accounting for 238 (87\%) of the 274 exclusions.

Included patients were predominantly elderly women, and the frequency of admission to medical and surgical services was similar between the groups (Table 1). Baseline characteristics were comparable, with the exception of antibiotic exposure within 7 days before collection of the urine, which was significantly more common among patients with a negative culture result. The following signs and symptoms were present in at least $10 \%$ of patients in each group: abnormalities in the

Table 1. Baseline Characteristics of Study Population*

\begin{tabular}{|c|c|c|c|c|}
\hline Characteristic & $\begin{array}{l}\text { All Patients } \\
\quad(n=80)\end{array}$ & $\begin{array}{l}\text { Positive Result } † \\
\quad(n=40)\end{array}$ & $\begin{array}{l}\text { Negative Resultt } \\
\quad(n=40)\end{array}$ & $p$ Value \\
\hline$\overline{\text { Age (years), mean }}$ & 71.4 (SD 17.1) & 73.2 (SD 18.6) & 69.6 (SD 15.4) & 0.36 \\
\hline Sex, no. female & $58(73)$ & $32(80)$ & $26(65)$ & 0.21 \\
\hline Duration of stay (days), median & $11.5(2-154)$ & $14.0(4-100)$ & $7.5(2-154)$ & 0.05 \\
\hline \multicolumn{5}{|l|}{ Admitting service } \\
\hline Medicine & 39 (49) & $20(50)$ & $19(48)$ & $>0.99$ \\
\hline Surgery & $41(51)$ & $20(50)$ & $21(53)$ & $>0.99$ \\
\hline $\begin{array}{l}\text { Admission due to actual or } \\
\text { suspected infection }\end{array}$ & $19(24)$ & $8(20)$ & $11(28)$ & 0.60 \\
\hline $\begin{array}{l}\text { No. of comorbidities per } \\
\text { patient, median }\end{array}$ & $3(0-6)$ & $3(0-5)$ & $3(0-6)$ & 0.56 \\
\hline $\begin{array}{l}\text { Exposure to antibiotics within } \\
7 \text { days before urine collection }\end{array}$ & $42(53)$ & $15(38)$ & $27(68)$ & 0.014 \\
\hline $\begin{array}{l}\text { Around-the-clock } \\
\text { antipyretic use }\end{array}$ & $14(18)$ & $6(15)$ & $8(20)$ & 0.77 \\
\hline $\begin{array}{l}\text { Duration of catheterization } \\
\text { at time of urine culture, } \\
\text { median (days) }\end{array}$ & $1.47(0.01-35.51)$ & $1.74(0.04-35.51)$ & $1.33(0.01-11.17)$ & 0.56 \\
\hline
\end{tabular}

SD = standard deviation.

*Data reported as median (range) or number (percent), unless otherwise specified.

†"Result" refers to results of urine culture.

\#For comparison of patients with positive and negative culture results.

IThe insertion date was not available for $16(20 \%)$ of the 80 patients: 6 of 40 with positive culture result and 10 of 40 with negative culture result. For these patients, the day of admission was considered day 1 of catheterization. 
leukocyte count, altered mental status, temperature instability, gastrointestinal complaints, malaise or lethargy, appearance and/or smell of the urine, abnormalities in urine flow (i.e., incontinence, frequency, urgency), and discomfort (i.e., dysuria, pain). Among the 40 patients with a positive culture result, 6 of the cultures involved 2 micro-organisms. Gram-negative organisms accounted for 31 (67\%) of the 46 micro-organisms cultured, and Escherichia coli was the most common species (14/46 [30\%]).

Antibiotic therapy was prescribed for a urinary indication in $40(50 \%)$ of the patients in the study population: 32 of the patients with a positive culture result and 8 of those with a negative culture result $(p<0.001)$. The indication was clearly documented in 33 of these 40 patients. For the remaining 7 patients, the indication was assigned according to a priori definitions $(n=4)$ or by the independent review panel $(n=3)$. Fluoroquinolones accounted for 25 (63\%) of the 40 final prescriptions and represented the most frequently prescribed class for patients in both groups. The median duration of therapy (and range) was 7 days (3-13 days) for the entire population.
Duration of therapy did not differ significantly between the positive and negative culture result groups ( 8 days [3-13 days] and 6.5 days [ $4-12$ days], respectively; $p=0.09$ ).

The primary end point, concordance, was observed for $63 \%$ of all patients and was significantly greater among patients with a negative culture result than among those with a positive result ( $85 \%$ versus $40 \%, p<0.001$; Table 2 ). The kappa statistic for the level of agreement between treatment decisions of the expert panel and prescribers was 0.25 (95\% confidence interval [CI] 0.09 to 0.41$)$ for the entire study population, 0.42 (95\% CI 0.06 to 0.79 ) for patients with a negative culture result, and 0.08 (95\% CI -0.07 to 0.22 ) for those with a positive result. Concordance was primarily determined by patients with a negative culture result for whom the expert panel deemed that therapy was not indicated and who did not receive antibiotics (31 of 34 patients) and patients with a positive result for whom the expert panel deemed that therapy was indicated and who did receive antibiotics ( 9 of 16 patients) (Table 2 ). For the 12 patients for whom therapy was indicated (as assessed by the expert panel) and received ( 9 with a positive result and 3 with a negative

Table 2. Concordance of Treatment Decisions of Expert Panel and Prescribers*

\begin{tabular}{|c|c|c|c|c|}
\hline Characteristic & $\begin{array}{l}\text { All Patients } \\
\quad(n=80)\end{array}$ & $\begin{array}{l}\text { Positive Resultt } \\
(n=40)\end{array}$ & $\begin{array}{c}\text { Negative Resultt } \\
(n=40)\end{array}$ & $p$ Value $\ddagger$ \\
\hline Concordant§ & $50(63)$ & $16(40)$ & $34(85)$ & $<0.001$ \\
\hline $\begin{array}{l}\text { Antibiotic indicated } \\
\text { and received }\end{array}$ & 12 & 9 & 3 & \\
\hline $\begin{array}{l}\text { Antibiotic not indicated and } \\
\text { not received }\end{array}$ & 38 & 7 & 31 & \\
\hline Discordant & $30(38)$ & $24(60)$ & $6(15)$ & Not tested \\
\hline $\begin{array}{l}\text { Antibiotic indicated and } \\
\text { not received }\end{array}$ & 2 & 1 & 1 & \\
\hline $\begin{array}{l}\text { Antibiotic not indicated } \\
\text { and received }\end{array}$ & 28 & 23 & 5 & \\
\hline
\end{tabular}

*Data reported as number of patients, with percentage in parentheses for the 2 main categories (concordant and discordant). †"Result" refers to results of urine culture.

\#For comparison of patients with positive and negative culture results.

$\S$ Concordance was the primary end point.

Table 3. Unnecessary Days of Antibiotic Therapy*

\begin{tabular}{|c|c|c|c|c|}
\hline Characteristic & $\begin{array}{l}\text { All Patients } \\
\quad(n=80)\end{array}$ & $\begin{array}{l}\text { Positive Resultt } \\
\quad(n=40)\end{array}$ & $\begin{array}{c}\text { Negative Result } \dagger \\
\quad(n=40)\end{array}$ & $p$ Value \\
\hline $\begin{array}{l}\text { UDT per inpatient-days of } \\
\text { therapy received }+ \\
\text { outpatient-days of therapy } \\
\text { prescribed }\end{array}$ & $201 / 293(69)$ & $175 / 240(73)$ & $26 / 53(49)$ & 0.001 \\
\hline $\begin{array}{l}\text { UDT per inpatient-days of } \\
\text { therapy received }\end{array}$ & 169/239 (71) & 147/197 (75) & $22 / 42(52)$ & 0.007 \\
\hline UDT per 1000 inpatient-days§ & 107 & 165 & 32 & $<0.001$ \\
\hline
\end{tabular}

UDT = unnecessary days of therapy.

*Data reported as number (percent), unless otherwise specified.

†"Result" refers to results of urine culture.

\#For comparison of patients with positive and negative culture results.

§No. of inpatient days was 1582 overall, consisting of 889 for patients with positive culture result and 693 for patients with negative culture result. 
result), the antibiotic prescribed was appropriate according to susceptibility results and the patient's allergy status.

The most common reason for discordance was the administration of antibiotics when therapy was not indicated, as assessed by the expert panel ( 23 of 24 patients with a positive result and 5 of 6 patients with a negative result; Table 2). This discordance contributed to a significantly greater number of unnecessary days of antibiotic therapy for the patients with a positive result when calculated as a percentage of total days of therapy (inpatient plus outpatient), when restricted to days of inpatient therapy only, and when standardized to 1000 inpatient-days (Table 3). It was determined that 201 (69\%) of 293 days of antibiotic therapy related to catheter urine cultures in the inpatient plus outpatient setting were unnecessary. When the analysis was restricted to the inpatient setting, the result was similar (169/239 [71\%]). Patients with positive culture results accounted for 175 (87\%) of the 201 unnecessary days of therapy in the inpatient plus outpatient setting. The result was the same (147/169 [87\%]) for the inpatient only setting. Discordance due to a failure to administer therapy when such therapy was indicated, as assessed by the expert panel, occurred in only 2 patients (Table 2), neither of whom experienced an adverse outcome.

The predefined 7-day assessment period was partially or fully completed during the hospital stay for $70(88 \%)$ of the 80 patients (36 with a positive result and 34 with a negative result). The other 10 patients were discharged before or on the day of the final culture report. During the assessment period, 3 patients ( 2 with a positive result and 1 with a negative result) were re-treated for a urinary indication, and 1 patient with a negative result who was not initially treated was started on antibiotic therapy for a urinary indication. Adverse events occurred in 2 of the 23 patients with a positive result whose therapy was deemed discordant because antibiotics were prescribed when not indicated. One of these patients experienced a diffuse rash after IV administration of ceftazidime, which was continued on an outpatient basis. The other patient experienced antibiotic-associated diarrhea with ciprofloxacin therapy. Two patients with a positive culture result were readmitted to hospital. The first readmission was due to the previously described ceftazidime-related rash. The second readmission, for a patient whose therapy was deemed concordant, was due to urinary symptoms after a 5-day course of trimethoprimsulfamethoxazole for a sensitive strain of Escherichia coli. The repeat urine culture grew E. coli resistant to this agent. Two patients with a positive culture result and concordant therapy died during the observation period for reasons unrelated to urinary tract infection.

\section{DISCUSSION}

This study assessed the appropriateness of antibiotic prescribing in response to the results of catheter urine cultures. The study design was unique because it included both patients with positive culture results and those with negative results and because a blinded expert panel was utilized to determine if treatment had in fact been indicated. The panel's assessment was based on the clinical scenario as opposed to reliance on guidelines, which are limited in their ability to address the complexity of individual cases typically encountered in an acute care setting. ${ }^{2}$ Patients receiving antibiotics within 7 days before the urine collection were eligible for inclusion in the study. This situation reflects a challenge commonly faced by prescribers who are interpreting urine culture results in the hospital setting, where antibiotic use is frequent and may sterilize the urine. ${ }^{9}{ }^{12-14}$ Dalen and others ${ }^{9}$ reported the concomitant use of antibiotics in $28 \%$ of their catheterized population at study enrolment. In comparison, $53 \%$ of the patients enrolled in the current study were receiving or had received antibiotics within 7 days before urine collection, with use of antibiotics being significantly more common among patients with a negative culture result. It is noteworthy that despite negative culture results, 4 (10\%) of 40 catheterized patients were deemed to have a urinary indication for antibiotic therapy, based on the expert panel's assessment of the clinical scenario. Three of these assessments were in agreement with the presciber's treatment decision, and the patients received therapy when indicated.

Appropriate prescribing, as determined by concordance in treatment decisions between the expert panel and prescribers, was significantly lower among patients with a positive culture result than among those with a negative result. Discordance was predominantly the result of antibiotics being prescribed when such therapy was not indicated and was greatest among patients with a positive culture result (23 [58\%] of 40 patients). This figure exceeds the rates of $32 \%$ and $52 \%$ previously reported for inappropriate prescribing of antimicrobials in catheterized patients with asymptomatic bacteriuria ${ }^{9,10}$; this discrepancy may be related to differences in study design.

The kappa statistics for concordance of treatment decisions revealed poor agreement for patients with a positive culture result, moderate agreement for those with a negative result, and fair agreement overall. Although the wide confidence intervals indicate that further research is necessary, these results provide preliminary evidence suggesting the need to improve prescribing for all patients with urine cultures from indwelling catheters, whether the results are positive or negative. Educational efforts may be effective in reducing the unnecessary use of antibiotic therapy in response to urine culture results. ${ }^{8,15}$

Although the unnecessary use of antimicrobials related to catheter urine cultures has been previously described, it has not been well quantified. ${ }^{9} 10$ The current analysis determined that approximately $70 \%$ of antibiotic days related to catheter urine cultures were unnecessary and that patients with a positive culture result accounted for $87 \%$ of those unnecessary days of 
therapy. The number of unnecessary days of therapy remained significantly greater for patients with a positive result than for those with a negative result after standardization to 1000 inpatient-days, which supports the need for an antimicrobial stewardship initiative.

The unnecessary use of antibiotics may result in unintended adverse events, antibiotic resistance, or superinfection, as well as increased health care costs..$^{10,15-20}$ Accompanying the greater proportion of unnecessary antibiotic days in the patients with a positive culture result was the observation that adverse effects occurred in 2 of 23 patients whose therapy was deemed discordant because antibiotics were prescribed when not indicated. One of these adverse effects resulted in readmission to hospital.

This study had several limitations. The retrospective design limited the completeness of data collection related to outcomes and duration of catheterization. The lack of informed consent restricted the assessment of predefined outcomes to data available through hospital records and the Ontario Drug Benefit profile viewer. Restrictive inclusion criteria were used to enrol patients who were immunocompetent and not critically ill, to facilitate decision-making about the indication for treatment; however, these criteria limit the generalizability of findings to hospitalized patients with relatively uncomplicated medical situations. The possibility of unintended bias in collecting and presenting the data to the expert panel was minimized by having a single investigator use a predefined structured format and a priori definitions. Although the sample size was relatively small, statistically and clinically significant differences were observed for the primary end point of concordance with respect to the need for therapy and the secondary end point of unnecessary days of antibiotic consumption.

\section{CONCLUSIONS}

Appropriate antibiotic prescribing was more common among patients with negative urine culture results than among those with positive results. However, these data provide preliminary evidence suggesting an opportunity to improve prescribing in both groups. Unnecessary days of therapy and adverse effects were more commonly observed in patients with positive results. Stewardship initiatives should focus on the indications for treatment and increasing awareness of the risks associated with unnecessary antibiotic consumption, including adverse events and the development of antibiotic resistance.

\section{References}

1. Warren JW. Catheter-associated urinary tract infections. Int J Antimicrob Agents. 2001;17(4):299-303.

2. Hooton TM, Bradley SF, Cardenas DD, Colgan R, Geerlings SE, Rice JC, et al.; Infectious Diseases Society of America. Diagnosis, prevention, and treatment of catheter-associated urinary tract infection in adults: 2009 international clinical practice guidelines from the Infectious Diseases Society of America. Clin Infect Dis. 2010;50(5):625-63.

3. Tambyah PA, Maki DG. Catheter-associated urinary tract infection is rarely symptomatic: a prospective study of 1,497 catheterized patients. Arch Intern Med. 2000;160(5):678-82.

4. Garibaldi RA, Burke JP, Dickman ML, Smith CB. Factors predisposing to bacteriuria during indwelling urethral catheterization. $N$ Engl J Med. 1974;291(5):215-9.

5. Platt R, Polk BF, Murdock B, Rosner B. Risk factors for nosocomial urinary tract infection. Am J Epidemiol. 1986;124(6):977-85.

6. Saint S. Clinical and economic consequences of nosocomial catheterrelated bacteriuria. Am J Infect Control. 2000;28(1):68-75.

7. Silver SA, Baillie L, Simor AE. Positive urine cultures: a major cause of inappropriate antimicrobial use in hospitals? Can J Infect Dis Med Microbiol. 2009;20(4):107-11.

8. Pavese P, Saurel N, Labarère J, Decouchon C, Vittoz JP, Foroni L, et al. Does an educational session with an infectious diseases physician reduce the use of inappropriate antibiotic therapy for inpatients with positive urine culture results? A controlled before-and-after study. Infect Control Hosp Epidemiol. 2009;30(6):596-9.

9. Dalen DM, Zvonar RK, Jessamine PG. An evaluation of the management of asymptomatic catheter-associated bacteriuria and candiduria at The Ottawa Hospital. Can J Infect Dis Med Microbiol. 2005;16(3):166-70.

10. Cope M, Cevallos ME, Cadle RM, Darouiche RO, Musher DM, Trautner BW. Inappropriate treatment of catheter-associated asymptomatic bacteriuria in a tertiary care hospital. Clin Infect Dis. 2009;48(9):1182-8.

11. Altman DG. Practical statistics for medical research. London (UK): Chapman and Hall; 1991.

12. Gandhi T, Flanders SA, Markovitz E, Saint S, Kaul DR. Importance of urinary tract infection to antibiotic use among hospitalized patients. Infect Control Hosp Epidemiol. 2009;30(2):193-5.

13. Riley DK, Classen DC, Stevens LE, Burke JP. A large randomized clinical trial of a silver-impregnated urinary catheter: lack of efficacy and staphylococcal superinfection. Am J Med. 1995;98(4):349-56.

14. Hustinx WN, Mintjes-de Groot AJ, Verkooyen RP, Verbrugh HA. Impact of concurrent antimicrobial therapy on catheter-associated urinary tract infection. J Hosp Infect. 1991;18(1):45-56.

15. Linares LA, Thornton DJ, Strymish J, Baker E, Gupta K. Electronic memorandum decreases unnecessary antimicrobial use for asymptomatic bacteriuria and culture-negative pyuria. Infect Control Hosp Epidemiol. 2011;32(7):644-8.

16. Warren JW, Anthony WC, Hoopes JM, Muncie HL Jr. Cephalexin for susceptible bacteriuria in afebrile, long-term catheterized patients. JAMA. 1982;248(4):454-8.

17. Hecker MT, Aron DC, Patel NP, Lehmann MK, Donskey CJ. Unnecessary use of antimicrobials in hospitalized patients: current patterns of misuse with an emphasis on the antianaerobic spectrum of activity. Arch Intern Med. 2003;163(8):972-8.

18. Alling B, Brandberg A, Seeberg S, Svanborg A. Effect of consecutive antibacterial therapy on bacteriuria in hospitalized geriatric patients. Scand J Infect Dis. 1975;7(3):201-7.

19. Nicolle LE, Bradley S, Colgan R, Rice JC, Schaeffer A, Hooton TM; Infectious Diseases Society of America; American Society of Nephrology; American Geriatric Society. Infectious Diseases Society of America guidelines for the diagnosis and treatment of asymptomatic bacteriuria in adults. Clin Infect Dis. 2005;40(5):643-54. Erratum: Clin Infect Dis. 2005;40(10):1556.

20. Gross PA, Patel B. Reducing antibiotic overuse: a call for a national performance measure for not treating asymptomatic bacteriuria. Clin Infect Dis. 2007;45(10):1335-7.

Jonathan Chiu, BScPhm, was at the time of this study, a Pharmacy Resident, Pharmacy Services, London Health Sciences Centre, London, Ontario. He is now a Critical Care Pharmacist in the Pharmacy Department, The Credit Valley Hospital and Trillium Health Centre, Mississauga, Ontario. 
G William Thompson, MD, is Associate Professor of Medicine, Division of Infectious Diseases, Department of Internal Medicine, Schulich School of Medicine and Dentistry, University of Western Ontario, London, Ontario.

Thomas W Austin, MD, is Professor Emeritus, University of Western Ontario, London, Ontario.

Zafar Hussain, MD, was, at the time of this study, Professor and Section Head, Department of Pathology and Laboratory Medicine, London Health Sciences Centre and Schulich School of Medicine and Dentistry, University of Western Ontario, London, Ontario. He is now Professor Emeritus with the University of Western Ontario.

Michael John, MB, ChB, is Medical Director, Infection Control, Department of Pathology and Laboratory Medicine, London Health Sciences Centre and Schulich School of Medicine and Dentistry, University of Western Ontario, London, Ontario.

Anne Marie Bombassaro, BScPhm, PharmD, is an Infectious Diseases Pharmacist, Pharmacy Services, London Health Sciences Centre, and Adjunct Assistant Professor, Department of Medicine, Schulich School of Medicine and Dentistry, University of Western Ontario, London, Ontario. She is also Assistant Professor (Status Only), Leslie Dan Faculty of Pharmacy, University of Toronto, Toronto, Ontario.

\section{Appendix 1. Exclusion criteria}

\section{Part 1}

- Candiduria

- Mixed bacterial growth (more than 2 micro-organisms)

- Emergency department visit (without admission)

- Admission to

- Intensive care unit (critical care trauma centre)

- Urology

- Nephrology

- Immunocompromising condition:

- Active or suspected malignancy

- HIV

- Transplantation
Sarah E Connelly, BSCPhm, MSc, was, at the time of this study, an Evidence-Based Medicine Pharmacist, Pharmacy Services, London Health Sciences Centre, London, Ontario.

Sameer Elsayed, MD, is Associate Professor of Medicine, Division of Infectious Diseases, Department of Internal Medicine, Schulich School of Medicine and Dentistry, and Department of Microbiology and Immunology, University of Western Ontario, London, Ontario.

Competing interests: None declared.

Address correspondence to:

Dr Anne Marie Bombassaro

London Health Sciences Centre

Victoria Hospital, Office B1-424

800 Commissioners Road E

London ON N6A 5W9

e-mail: annemarie.bombassaro@|hsc.on.ca

\section{Acknowledgements}

The authors express their gratitude for statistical support to Jamie A Seabrook, Department of Paediatrics, University of Western Ontario, and Children's Health Research Institute, Children's Hospital, London Health Sciences Centre, London, Ontario.

\section{Part 2}

- Pregnancy

- Spinal cord injury

- Underlying renal or urologic abnormalities, dialysis

- Involvement of infectious diseases department (admission to or consultation by)

- Immunosuppressive medication:

- Long-term steroids (defined a priori as uninterrupted steroid therapy for a period of at least 90 days at a daily prednisone dose of $\geq 10 \mathrm{mg} /$ day, a cumulative prednisone dose of $>700 \mathrm{mg}$, or equivalent doses of an alternative steroid formulation)

- Cancer chemotherapy

- Antirejection agents (e.g., azathioprine, cyclosporine, tacrolimus, sirolimus, mycophenolate)

- Monoclonal antibodies

- Methotrexate

Appendix 2. A priori definitions for prescriber treatment decisions when chart documentation was lacking

1. Prescriptions for norfloxacin or nitrofurantoin were interpreted as treatment received for a urinary indication.

2. Prescriptions for clindamycin, cloxacillin, macrolides, chloramphenicol, metronidazole, and oral vancomycin were interpreted as treatment received for a nonurinary indication.

3. Prescriptions of antibiotics other than those specified in 1 and 2 above were interpreted as follows:

a. Treatment was deemed to be for a non-urinary indication if the urine culture result was negative and another indication or culture result was present. b. Treatment was deemed to be for a urinary indication if the urine culture result was positive and another indication or culture result was absent.

c. Treatment for a urinary indication was undetermined if the urine culture result was positive and another indication or culture result was present.

d. Treatment for a urinary indication was undetermined if the urine culture result was negative and another indication or culture result was absent. 